\title{
Comparison of Deconvolution-Based and Absorption Modeling IVIVC for Extended Release Formulations of a BCS III Drug Development Candidate
}

\author{
Filippos Kesisoglou, ${ }^{1,3}$ Binfeng Xia, ${ }^{1}$ and Nancy G. B. Agrawal ${ }^{2}$
}

Received 23 June 2015; accepted 5 August 2015; published online 20 August 2015

\begin{abstract}
In vitro-in vivo correlations (IVIVC) are predictive mathematical models describing the relationship between dissolution and plasma concentration for a given drug compound. The traditional deconvolution/convolution-based approach is the most common methodology to establish a level A IVIVC that provides point to point relationship between the in vitro dissolution and the in vivo input rate. The increasing application of absorption physiologically based pharmacokinetic model (PBPK) has provided an alternative IVIVC approach. The current work established and compared two IVIVC models, via the traditional deconvolution/convolution method and via absorption PBPK modeling, for two types of modified release (MR) formulations (matrix and multi-particulate tablets) of MK-0941, a BCS III drug development candidate. Three batches with distinct release rates were studied for each formulation technology. A two-stage linear regression model was used for the deconvolution/convolution approach while optimization of the absorption scaling factors (a model parameter that relates permeability and input rate) in Gastroplus ${ }^{\mathrm{TM}}$ Advanced Compartmental Absorption and Transit model was used for the absorption PBPK approach. For both types of IVIVC models established, and for either the matrix or the multiparticulate formulations, the average absolute prediction errors for AUC and $C_{\max }$ were below $10 \%$ and $15 \%$, respectively. Both the traditional deconvolution/convolution-based and the absorption/PBPK-based level A IVIVC model adequately described the compound pharmacokinetics to guide future formulation development. This case study highlights the potential utility of absorption PBPK model to complement the traditional IVIVC approaches for MR products.
\end{abstract}

KEY WORDS: absorption modeling; deconvolution; in vitro-in vivo correlation (IVIVC); modified release; physiologically based pharmacokinetic (PBPK) modeling.

\section{INTRODUCTION}

In vitro-in vivo correlations (IVIVCs) are defined as predictive mathematical models describing the relationship between dissolution and plasma concentration for a given drug (1). Since an IVIVC allows the formulator to project the impact of a formulation/manufacturing change on the product clinical performance, the establishment of IVIVC is highly desirable during formulation development and can be considered a very important component of quality by design. Especially for extended release (ER) products, where formulation composition is critical to controlling the input of the drug in the systemic circulation, IVIVCs are routinely sought after during drug development.

Available Regulatory Agency guidance details the different IVIVC levels, the methodologies for their establishment, and their regulatory application (1-3). These have also

\footnotetext{
${ }^{1}$ Biopharmaceutics, Pharmaceutical Sciences and Clinical Supply, Merck \& Co. Inc., WP75B-210, West Point, Pennsylvania 19486, USA.

${ }^{2}$ Pharmacokinetics, Pharmacodynamics and Drug Metabolism, Merck \& Co. Inc., West Point, Pennsylvania 19486, USA.

${ }^{3}$ To whom correspondence should be addressed. (e-mail: filippos_kesisoglou@merck.com)
}

been reviewed extensively in the literature (4-6). The establishment of point-to-point correlations (level A IVIVC) is seen as the most desirable and most informative correlation. The most common methodology for establishment of IVIVCs is the traditional deconvolution-based approach. However, alternative approaches including convolutionbased models can be considered as potential alternatives. The potential benefits of these approaches have also been extensively discussed in the literature $(7,8)$.

In the recent years, the development of absorption/ physiologically based pharmacokinetic model (PBPK) models has provided additional tools to link compound/formulation information to clinical pharmacokinetics (9). Oral Absorption/PBPK modeling involves the modeling of the key processes that dictate oral absorption, namely dissolution (which for controlled release formulations would be the release out of the formulation) and gut wall permeation (dictated by compound properties), to estimate the time course of absorption for a given compound/formulation. When combined with systemic drug distribution and elimination parameters, a prediction of plasma concentration profiles becomes possible. Availability of commercial software such as GastroPlus (10), Simcyp (11), PK-Sim (12), or Intellipharm (13) has facilitated adoption of these tools during formulation development. 
Given the ability of oral absorption/PBPK models to project plasma concentration profiles based on in vitro data, they can also be used as an alternative method to establish an IVIVC (14). The traditional deconvolution/convolution method is more suitable for compounds with linear pharmacokinetics that are well-absorbed throughout the GI tract. While it is possible to utilize the same models via further model adjustments, such as use of time scaling/time shifting and absorption time cut-offs, for compounds with more complex absorption patterns, it has been recommended that the resulting models may require additional validation steps (15). Due to the flexibility to incorporate physiological absorption processes (such as regional dependent absorption due to either physicochemical properties or transporter involvement) as well as saturable metabolic components, absorption/PBPK model may provide more flexibility in establishing IVIVC for these compounds. On the other hand, the need for validation/justification of a larger set of input parameters may be seen as a limitation for the PBPK models and raise similar requirements of further model validation.

In this manuscript, we detail the efforts to establish IVIVC via both the traditional deconvolution/convolution method as well as via absorption modeling for ER formulations for MK-0941, a BCS III development candidate. MK0941 was shown preclinically (16), and confirmed by the observed clinical data as discussed in this manuscript, to exhibit regional dependent absorption with reduced bioavailability from the colon compared to oral administration. Two types of formulations, matrix tablets and multiparticulates in capsules, were characterized in vitro and evaluated in a clinical study. Due to the regiodependent absorption, establishment of traditional deconvolution/convolution-based models was challenging and required a two-stage absorption function to achieve reasonable prediction errors. The absorption modeling-based approach on the other hand was shown to be able to provide a link between dissolution data and plasma concentration profiles when the regional absorption properties were incorporated in the software and resulted in comparable predictions to the traditional IVIVC model.

\section{MATERIALS AND METHODS}

\section{Formulation Information and Dissolution Data}

Two types of formulations were evaluated in the clinical study as described before (16). Three HPMC-based matrix tablets, prepared by varying the levels of HPMC in the formulation to adjust the compound release rate, were manufactured. Tablets exhibited release rates of approximately T80 (Time to $80 \%$ dissolution) of 8,12 , and $16 \mathrm{~h}$. Three multiparticulate formulations, consisting of a drug containing core with a functional ethylcellulose-based sustained-release coating, were also manufactured. The coating level was varied to achieve release rates comparable to what was achieved for the three matrix formulations. Formulation details have been discussed in a previous publication (16).

Dissolution for the formulations studies was conducted in a USP II system at $100 \mathrm{rpm}$. Six tablets for each release rate were tested. Average dissolution data used for the IVIVC are presented in Fig. 1.

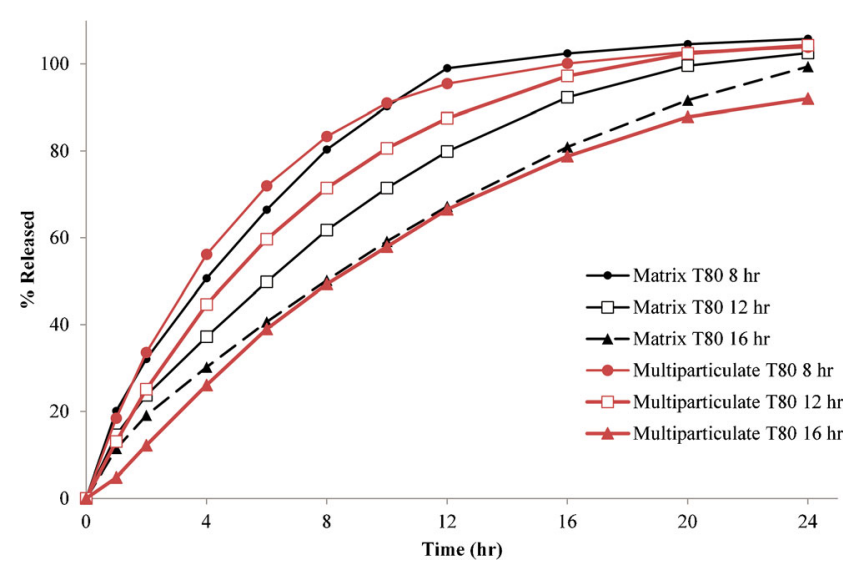

Fig. 1. Average $(n=6)$ dissolution profiles for matrix and multiparticulate MK-0941 formulations used for IVIVC model development

\section{Clinical Study Design}

PN003 was a phase I, two-part, single-dose study in healthy men and women. The formulations used for the exploration of the IVIVC reported herein were evaluated in part I of the study. Sixteen subjects were administered four of eight treatments in a randomized, crossover fashion. Treatments included placebo and 4-mg doses of the matrix and multiparticulate ER formulations or an immediate release (DFC IR) capsule formulation as a reference. The study was approved by Independent Institutional Review Board (IRB). Plasma sample analysis and pharmacokinetic calculations were conducted in a manner similar to what has been described before (16).

\section{Deconvolution/Convolution-Based IVIVC}

The deconvolution/convolution level A IVIVC analysis was conducted on the mean plasma concentration-plasma profiles in three steps, following the procedures in the respective FDA guidance (1). First, a unit impulse response (UIR) function was determined using the mean concentration-time data following the administration of the IR formulation; since study was only a partial crossover, individual UIR could not be calculated for all subjects within each treatment, which would be the typically preferred approach. Subsequently, the fraction of MK-0941 in vivo release as a function of time for each formulation was determined by deconvolution. Finally, an IVIVC relationship between in vitro and in vivo MK-0941 release for the formulations was fitted to the derived data. For the establishment of the correlation, the release data for each formulation were fit to the empirical Weibull function.

The initial model attempted was a traditional linear model with time scaling/shifting and with or without the inclusion of an absorption cut-off time (time after no further absorption takes place). However, the model resulted in large prediction errors (data not shown). Inspection of the time course of in vivo absorption indicated a change in the rate of absorption of the compound approximately $5-6 \mathrm{~h}$ after oral administration. Therefore, the linear model fails to capture the entirety of the absorption curve. To better describe the time-course of absorption and allow for a better IVIVC, a 
Table I. Summary of Input Parameters Used in the GastroPlus PBPK IVIVC Model of MK-0941

\begin{tabular}{|c|c|c|}
\hline Parameter & Value & Source \\
\hline Molecular weight (Dalton) & 460.5 & \\
\hline pH-dependent solubility $(\mathrm{mg} / \mathrm{mL})$ & & Experimental measurement (equilibrium solubility) \\
\hline $\mathrm{pH} 1.0$ & 2.17 & \\
\hline $\mathrm{pH} 2.0$ & 1.56 & \\
\hline $\mathrm{pH} 3.0$ & 1.47 & \\
\hline $\mathrm{pH} 4.0$ & 1.39 & \\
\hline $\mathrm{pH} 6.0$ & 0.79 & \\
\hline $\mathrm{pH} 7.0$ & 1.23 & \\
\hline $\mathrm{pH} 8.0$ & 1.24 & \\
\hline $\log \mathrm{D}_{\mathrm{pH}=7.4}$ & 1.64 & Experimental measurement \\
\hline \multirow{2}{*}{$\mathrm{pKa}$} & 0.91 (base) & ACD predictions \\
\hline & 12.3 (acid) & Experimental measurement: No Detectable pKa between 2.5 and 11 \\
\hline Mean precipitation time (s) & 900 & Default setting \\
\hline Dose $(\mathrm{mg})$ & 4 & From clinical study \\
\hline Unbound fraction in plasma (\%) & 65.2 & Experimental measurement \\
\hline Blood/plasma concentration ratio & 0.88 & Experimental measurement \\
\hline Absorption rate-constant $\left(k_{\mathrm{a}}\right)$ & 1.95 & Estimated from IR PK fit in PKPlus ${ }^{\circledR}$ \\
\hline LLC-PK1 $P_{\text {app }}\left(\times 10^{-6} \mathrm{~cm} / \mathrm{s}\right)$ & 9.0 & Converted to $1.5 \times 10^{-4} \mathrm{~cm} / \mathrm{s}$ human $P_{\text {eff }}$ as initial input \\
\hline Human $P_{\text {eff }}\left(\times 10^{-4} \mathrm{~cm} / \mathrm{s}\right)$ & 4 & Optimized by fitting oral IR PK data \\
\hline $\mathrm{CL} / \mathrm{F}(\mathrm{L} / \mathrm{h} / \mathrm{kg})$ & 0.420 & Estimated from IR PK fit in PKPlus ${ }^{\circledR}$ \\
\hline $\mathrm{V} / \mathrm{F}(\mathrm{L} / \mathrm{kg})$ & 2.67 & Estimated from IR PK fit in PKPlus ${ }^{\circledR}$ \\
\hline Elimination half-life (h) & 10.1 & Estimated from IR PK fit in PKPlus ${ }^{\circledR}$ \\
\hline$K_{12}\left(\mathrm{~h}^{-1}\right)$ & 0.170 & Estimated from IR PK fit in PKPlus ${ }^{\circledR}$ \\
\hline$K_{21}\left(\mathrm{~h}^{-1}\right)$ & 0.102 & Estimated from IR PK fit in PKPlus ${ }^{\circledR}$ \\
\hline$V_{2}(\mathrm{~L} / \mathrm{kg})$ & 1.67 & Estimated from IR PK fit in PKPlus ${ }^{\circledR}$ \\
\hline \multicolumn{3}{|l|}{ Absorption scaling factors (ASF) } \\
\hline \multicolumn{3}{|l|}{ Matrix/multiparticulate } \\
\hline Duodenum & $9.10 / 14.0$ & Optimized using optimization module as described in the \\
\hline Jejunum 1 & $5.20 / 4.8$ & MATERIALS AND METHODS section for matrix/multiparticulates formulations \\
\hline Jejunum 2 & $2.60 / 3.5$ & \\
\hline Ileum $1-3$ & $0.60 / 0.9$ & \\
\hline Caecum/colon & $0.026 / 0.05$ & \\
\hline
\end{tabular}

model with differential absorption scaling factors was developed as described earlier (15). The model is described by the following equations

For Time $<$ Tchange

Fabs $=$ AbsScale $1 \times$ Fdiss $($ Tscale $\times$ Time $)$

For Time $\geq$ Tchange

Fabs $=($ AbsScale $1-$ AbsScale 3$) \times$ Fdiss $($ Tscale $\times$ Tchange $)+$ AbsScale $2 \times$ Fdiss $($ Tscale $\times$ Time $)$

In the final model, a Tcutoff time parameter was also included. All parameters AbsScale1, AbScale2, Tscale, Tchange, and Tcutoff were estimated in the software and were not fixed.

The level A models were evaluated in accordance with the FDA criteria (1). The model-predicted parameters were compared to the corresponding observed values and the prediction error $(\% \mathrm{PE})$ was calculated according to:

$\% \mathrm{PE}=100 \times\left(\frac{\text { Predicted Parameter-Observed Parameter }}{\text { Observed Parameter }}\right)$.

All calculations were conducted in WinNonlin Phoenix (Certara USA, St. Louis, MO).

\section{Absorption Modeling-Based IVIVC}

The absorption modeling-based IVIVC was built using Advanced Compartmental Absorption and Transit (ACAT) model in GastroPlus (v8.0; Simulations Plus, Lancaster, CA). The structure of the model has been described in previous publications (9). Human PK parameters (volume of distribution: $\mathrm{V} / \mathrm{F}=2.67 \mathrm{~L} / \mathrm{kg}, \mathrm{CL} / \mathrm{F}=0.42 \mathrm{~L} / \mathrm{h} / \mathrm{kg}$ ) were obtained by fitting the oral data of immediate release (DFC-IR) capsule using a two compartmental pharmacokinetic model in PKPlus ${ }^{\circledR}$ (Simulations Plus, Lancaster, CA). The physicochemical properties of MK-0941 are shown in Table I, including $\mathrm{pKa}, \operatorname{LogD}, \mathrm{pH}-$ dependent solubility. The effective permeability $\left(P_{\text {eff }}\right)$ value of MK-0941 was converted from an in-house Caco-2 permeability data using an internal conversion equation as the initial estimate (final parameters shown in Table I). The "CR: integral tablet" and "CR: Dispersed" were designated for the matrix-based tablet and multi-particulate (MP) tablet, respectively, according to their corresponding formulation disintegration patterns. The experimental in vitro dissolution profiles for all formulations (Fig. 1) were loaded as *.dsd files in ACAT model. The Weibull release functions were then used to fit the in vitro dissolution data and the resulting Weibull release profiles (regarded as dissolution profile) were further incorporated in the model as *.crd file. The default physiological Opt-logD model SA/V 6.1 was used to simulate the pharmacokinetic profile except the absorption 
Table II. Summary Pharmacokinetic Parameters for Formulations Tested in IVIVC Study (Mean and Standard Deviation Reported Except Tmax for Which Median and Range Are Reported)

\begin{tabular}{|c|c|c|c|c|c|}
\hline Treatment & $\begin{array}{l}\text { AUC } \\
(\mathrm{nM} \times \mathrm{h})\end{array}$ & $\begin{array}{l}C_{\max } \\
(\mathrm{nM})\end{array}$ & $\begin{array}{l}\mathrm{C} 12 \mathrm{hr} \\
(\mathrm{nM})\end{array}$ & $\begin{array}{l}\text { C24hr } \\
(\mathrm{nM})\end{array}$ & $\begin{array}{l}\text { Tmax } \\
(\mathrm{hr})\end{array}$ \\
\hline $\begin{array}{l}\text { Matrix } \\
\text { T80 } 8 \mathrm{~h}\end{array}$ & 188 (64) & $19(9)$ & $4.1(2.0)$ & $1.5(0.7)$ & $2(0.5-4)$ \\
\hline $\begin{array}{l}\text { Matrix } \\
\text { T80 } 12 \mathrm{~h}\end{array}$ & $192(53)$ & $13(4)$ & $3.5(1.3)$ & $2.9(1.7)$ & $2(1-4)$ \\
\hline $\begin{array}{l}\text { Matrix } \\
\text { T80 16 h }\end{array}$ & 149 (96) & $10(4)$ & $2.5(1.3)$ & $1.7(1.4)$ & $1.5(0.5-4)$ \\
\hline $\begin{array}{l}\text { Multiparticulate } \\
\text { T80 } 8 \mathrm{~h}\end{array}$ & $212(88)$ & $20(8)$ & $5.3(2.2)$ & $1.9(0.8)$ & $2(2-6)$ \\
\hline $\begin{array}{l}\text { Multiparticulate } \\
\text { T80 } 12 \mathrm{~h}\end{array}$ & $206(85)$ & $13(5)$ & $4.2(2.1)$ & $1.9(0.7)$ & $2(1-4)$ \\
\hline $\begin{array}{l}\text { Multiparticulate } \\
\text { T80 } 16 \text { h }\end{array}$ & $156(64)$ & $8(3)$ & $4.1(2.3)$ & $1.7(0.8)$ & $4(2-10)$ \\
\hline IR DFC & 288 (74) & $101(48)$ & $3.5(0.6)$ & $1.1(0.5)$ & $1(0.5-2)$ \\
\hline
\end{tabular}

scaling factor (ASF). In Gastroplus ACAT model, rate of drug absorption is the product of effective absorption rate coefficient (ka), unbound drug concentration difference in lumen and enterocyte $\left(C_{\text {lumen }}-C_{\text {ent,u }}\right)$, and the fluid volume in each compartment, where ka is the product of $P_{\text {eff }}$ and ASF for that compartment. ASF is a multiplier accounting for variations in surface-to-volume ratio, $\mathrm{pH}$ effects, and other absorption-ratedetermining effects that differ from one compartment to another. Since the product of ASF and $P_{\text {eff }}$ dictates the absorption rate (ka) of compound in solution, optimization of either one would be an option for modeling regiodependent permeability. For the absorption modeling-based IVIVC, a 1:1 relationship between in vitro and in vivo dissolution was assumed. The assumption is made to allow for fitting of a single set of parameters (ASFs). It is acknowledged, and will be discussed later in the manuscript, that alternative approaches to developing the model may be possible where the in vitro-in vivo relationship may deviate from 1:1. Subsequently, ASF values in duodenum, jejunum1, jejunum2, ileum, and colon were simultaneously estimated (instead of using the default value physiological Opt-logD model SA/V 6.1) by fitting to the observed concentration versus time curves of either formulation technology. Initially, optimization was conducted manually by adjusting the ASF values until a reasonable description of the plasma concentration profile was obtained. At that point, the ASF values were used as initial estimates for further fitting using the Optimization module in the software (objective function weight was set as $1 / \mathrm{Y}^{\wedge} 2$ and the concentration-time profile was used as the observations weight). The optimization will be considered successful if the point estimation (e.g., mean $\left.C_{\max }, \mathrm{AUC}\right)$ of internal validation dataset was within $15 \%$ of the observed values, similarly to what is outlined in the relevant FDA guidance (1). The resulted simulated concentration versus time curves were compared with the observed data and the point estimates were also tested based on the criteria.

\section{RESULTS}

\section{Clinical Pharmacokinetics}

Administration of the ER formulations in the fasted state resulted qualitatively in the desired profile, with somewhat blunted and delayed $C_{\max }$ and concentrations sustained for longer than those with the IR formulation. In the fasted state, the performance of the matrix and multiparticulate ER formulations was similar at each of the release rates. Relative to the IR formulation, geometric mean $C_{\max }$ ratios were 0.20 , 0.13 , and 0.10 for the 8,12 , and 16 -h release rates, respectively, for the matrix ER formulation and $0.21,0.13$, and 0.08 , respectively, for the multiparticulate formulation. However, bioavailability of the ER formulations is reduced as compared to the IR formulation (most substantially for the 16-h release rate formulations), suggesting that absorption may only be modest in the colon which is in line with the dog colonic absorption data. Relative to the IR formulation, geometric mean AUC ratios were 0.64, 0.67, and 0.45 for the 8,12 , and $16-\mathrm{h}$ release rates, respectively, for the matrix ER formulation and $0.70,0.67$, and 0.51 , respectively, for the multiparticulate formulation. As a result of the lower bioavailability, the $\mathrm{C} 12 \mathrm{hr}$ values for the ER formulations were not notably different from those of the IR formulation. However, C24hr values for the ER formulations were higher than those for the IR formulation, consistent with the sustained concentrations with the ER formulations. In

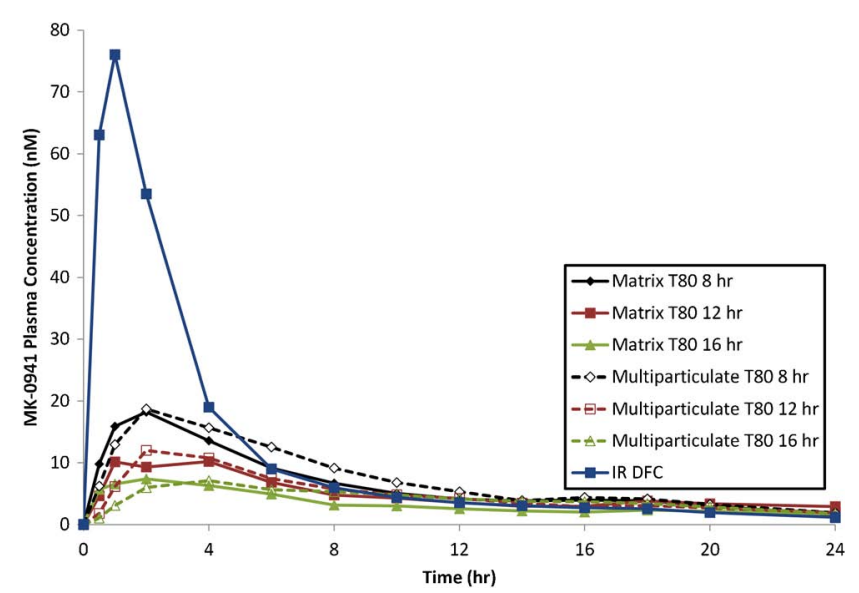

Fig. 2. Mean $(n=16)$ plasma concentration vs. time profiles for MK0941 IR and ER formulations 


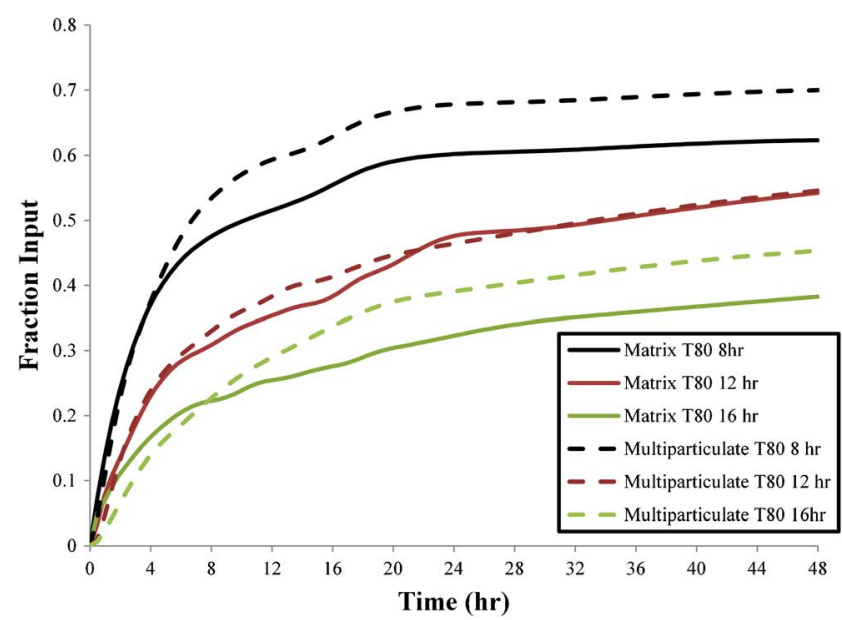

Fig. 3. Average fraction input vs. time profiles for MK-0941 ER formulations estimated by deconvolution

addition, as typically expected, PK variability was generally greater for the ER formulations than the IR formulation, as suggested by the higher \% CV in PK parameter values for the ER formulations. Summary pharmacokinetic data are summarized in Table II while average plasma concentration $v s$. time profiles are shown in Fig. 2.

\section{Deconvolution/Convolution-Based IVIVC}

The time course of absorption estimated via deconvolution is shown in Fig. 3. The parameters for the final model developed are shown in Table III for the matrix and multiparticulate formulations. In the case of the matrix formulations, while the model reasonably predicted the pharmacokinetic data for the fast and slow formulation, it over-predicted the exposure of the slow formulation, with a PE $>15 \%$ (Table IV). The multiparticulate formulation-based model accurately predicted the pharmacokinetic data for all formulations, with acceptable prediction errors. Figure 4 shows the observed and predicted plasma concentration $v s$. time data for each model (only predictions within respective formulation type shown).

As an additional exploratory step, the model developed for each of the matrix or multiparticulate systems was used to project exposure of the other system. These data are included in Table IV. While some deviations on the projections of the "external" system are observed, in general the behavior of the compound is described reasonably well taking into consideration the expected different behavior of each release system; most commonly IVIVCs are attempted within same formulation technology.

\section{Absorption Modeling-Based IVIVC}

The input function of drug release kinetics were found best characterized using the single-phase Weibull function by fitting the in vitro dissolution data for each formulation (Fig. 5). The optimized ASF values in GI compartment ranged from 0.026 to 9.1 and from 0.050 to 14.0 for matrix tablets and multiparticulates formulation, respectively (Table I), and were found higher in upper intestine (e.g., duodenum) and gradually decreased in lower part of intestine (e.g., caecum and large intestine). The baseline permeability value in the model was $4 \times 10^{-4} \mathrm{~cm} / \mathrm{s}$. It is acknowledged that the value is higher than what would be considered a BCS III compound if one was to assume constant permeability throughout the intestine; for the MK-0941 model, the absorption rate is significantly diminished past jejunum given the lower ASF values. The trend of ASF decreasing suggested a decreased absorption rate when CR formulations moved along to the lower intestine regions. The finding is in line with the region-dependent absorption of the compound in dogs where wherein intra-colonic administration of a solution formulation resulted in $40 \%$ bioavailability relative to oral administration (16). Although the shorter transit time has been highlighted in the past as a limitation for evaluation of modified release dosage forms, it has been suggested that the dog represents a reasonable model for evaluation of colonic relative bioavailability after solution administration (17). The simulated plasma concentration vs. time curves were generally in agreement with the observed clinical results for matrix-based and multiparticulates tablet (Fig. 6). The point estimates and deviation of prediction using PBPKIVIVC model for matrix and multiparticulates tablet are summarized in Table $\mathrm{V}$. The average of deviation of point estimates for AUC and $C_{\max }$ were 1.8 and $11.0 \%$, respectively, which is in line with the acceptable criteria for IVIVC model validation (1).

\section{DISCUSSION}

The use of IVIVCs for regulatory applications is well documented in the literature and in relevant Regulatory Agency guidance (1-3). However, application of IVIVC is not confined only in the late-stage development and regulatory space. Formulation composition and process changes are commonplace during drug product development as a drug candidate progresses from early to late stage clinical trials. For formulation and biopharmaceutics scientists,

Table III. IVIVC Model Parameters for Traditional Deconvolution/Convolution Approach

\begin{tabular}{lcr}
\hline & \multicolumn{1}{c}{ Matrix model } & Multiparticulate model \\
\hline Parameter & Parameter estimate (\% CV) & Parameter estimate (\% CV) \\
\hline AbsScale1 & $1.45(7.2 \%)$ & $1.08(3.7 \%)$ \\
AbsScale2 & $0.32(4.6 \%)$ & $0.33(4.1 \%)$ \\
Tscale & $0.35(7.4 \%)$ & $0.50(3.7 \%)$ \\
Tchange & $5.12(4.7 \%)$ & $5.37(7.1 \%)$ \\
Tcutoff & $33.9(3.9 \%)$ & $44.1(14.1 \%)$ \\
\hline
\end{tabular}



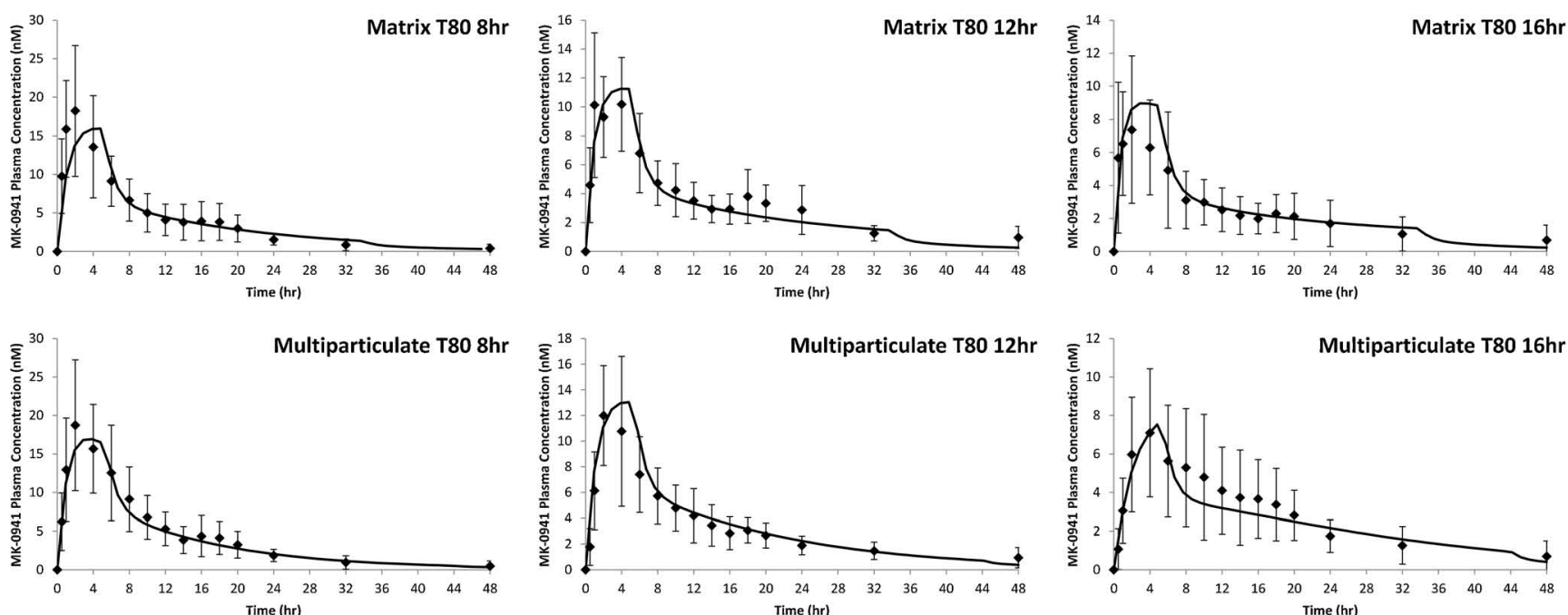

Fig. 4. Traditional deconvolution/convolution IVIVC model predicted (lines) vs. observed (diamonds, mean \pm SD) plasma concentration $v s$. time profiles for MK-0941 ER formulation

understanding the clinical impact of these changes is critical to enabling the design and development of a successful pharmaceutical product. For modified release (MR) products, the composition of the formulation is specifically intended to provide a targeted input rate that optimizes the compound pharmacokinetics. Thus the ability to use dissolution data and translate them via an IVIVC to a clinical pharmacokinetic outcome can significantly simplify the formulation development process. For the MK-0941 case study presented here, the study represented the initial introduction of a MR formulation in the clinic. The study was designed with an IVIVC in mind to guide future formulation development.

At a dose of $4 \mathrm{mg}$, MK-0941 is a BCS Class III compound based on previously reported low-moderate cell line permeability (Table I) (16). However, bioavailability in preclinical species was moderate to high $(48 \%$ in rats and $100 \%$ in dogs at doses $0.3-3 \mathrm{mg} / \mathrm{kg}$ ), indicating compound is reasonably well-absorbed in vivo. In the absence of conclusive in vivo data indicating high permeability and with the lower bioavailability in the rats, the designation of BCS III is maintained for the purposes of this manuscript. The compound exhibited regional dependent absorption with relative bioavailability of $40 \%$ after intra-colonic administration (relative to oral administration). There is no specific mechanism that the regional dependent absorption is attributed to and it is believed to, at least partially, be a reflection of the compound moderate lipophilicity $\left(\log \mathrm{D}_{\mathrm{pH} 7.4}=1.64\right)$ and the lower absorptive area in the colon. The compound is a Pgp substrate (BAAB ratio of 16.1 in human MDR1 transfected LLCPK1; experiment conducted at 5 - $\mu \mathrm{M}$ concentration) but demonstrated moderate-high bioavailability in preclinical species and linear PK across a dose range of 1$6 \mathrm{mg}$ tested in the single ascending dose study; thus it is unclear if P-gp is affecting oral absorption. Given the observed region-dependent absorption MK-0941 represented an interesting model compound to attempt a comparison of

Table IV. Prediction errors of AUC (in $\mathrm{nM} \times \mathrm{h})$ and $C_{\max }($ in $\mathrm{nM})$ for Traditional Deconvolution/Convolution IVIVC Model

\begin{tabular}{|c|c|c|c|c|c|c|c|}
\hline \multirow[b]{2}{*}{ Formulations } & \multirow[b]{2}{*}{ Parameters } & \multirow{2}{*}{$\frac{\text { Matrix model }}{\text { Pred. }}$} & \multicolumn{5}{|c|}{ Multiparticulate model } \\
\hline & & & Obs. & $\% \mathrm{PE}$ & Pred. & Obs. & $\% \mathrm{PE}$ \\
\hline \multirow[t]{2}{*}{ Matrix $8 \mathrm{~h}$} & AUC & 180 & 176 & 2.26 & 184 & 176 & 4.82 \\
\hline & $C_{\max }$ & 15.9 & 18.2 & -12.7 & 15.8 & 18.2 & -13.6 \\
\hline \multirow[t]{2}{*}{ Matrix $12 \mathrm{~h}$} & AUC & 141 & 152 & -7.79 & 153 & 152 & 0.22 \\
\hline & $C_{\max }$ & 11.3 & 10.2 & 10.5 & 11.1 & 10.2 & 9.16 \\
\hline \multirow[t]{2}{*}{ Matrix $16 \mathrm{~h}$} & AUC & 117 & 106 & 10.6 & 132 & 106 & 24.8 \\
\hline & $C_{\max }$ & 8.93 & 7.38 & 21.1 & 8.72 & 7.38 & 18.2 \\
\hline \multirow[t]{2}{*}{ Average } & AUC & N/A & N/A & 6.87 & N/A & N/A & 9.94 \\
\hline & $C_{\max }$ & N/A & N/A & 14.7 & N/A & N/A & 13.6 \\
\hline \multirow[t]{2}{*}{ Multiparticulate $8 \mathrm{~h}$} & AUC & 188 & 200 & -6.05 & 189 & 200 & -5.47 \\
\hline & $C_{\max }$ & 17.2 & 18.7 & -8.10 & 16.9 & 18.7 & -10.0 \\
\hline \multirow[t]{2}{*}{ Multiparticulate $12 \mathrm{~h}$} & AUC & 155 & 152 & 1.85 & 165 & 152 & 8.37 \\
\hline & $C_{\max }$ & 12.8 & 12.0 & 6.90 & 13.0 & 12.0 & 8.18 \\
\hline \multirow{2}{*}{ Multiparticulate $16 \mathrm{~h}$} & AUC & 99.0 & 127 & -21.8 & 118 & 127 & -7.15 \\
\hline & $C_{\max }$ & 6.50 & 7.10 & -8.43 & 7.10 & 7.10 & 0.09 \\
\hline \multirow[t]{2}{*}{ Average } & AUC & N/A & N/A & 9.91 & N/A & N/A & 7.00 \\
\hline & $C_{\max }$ & N/A & N/A & 7.81 & N/A & N/A & 6.09 \\
\hline
\end{tabular}



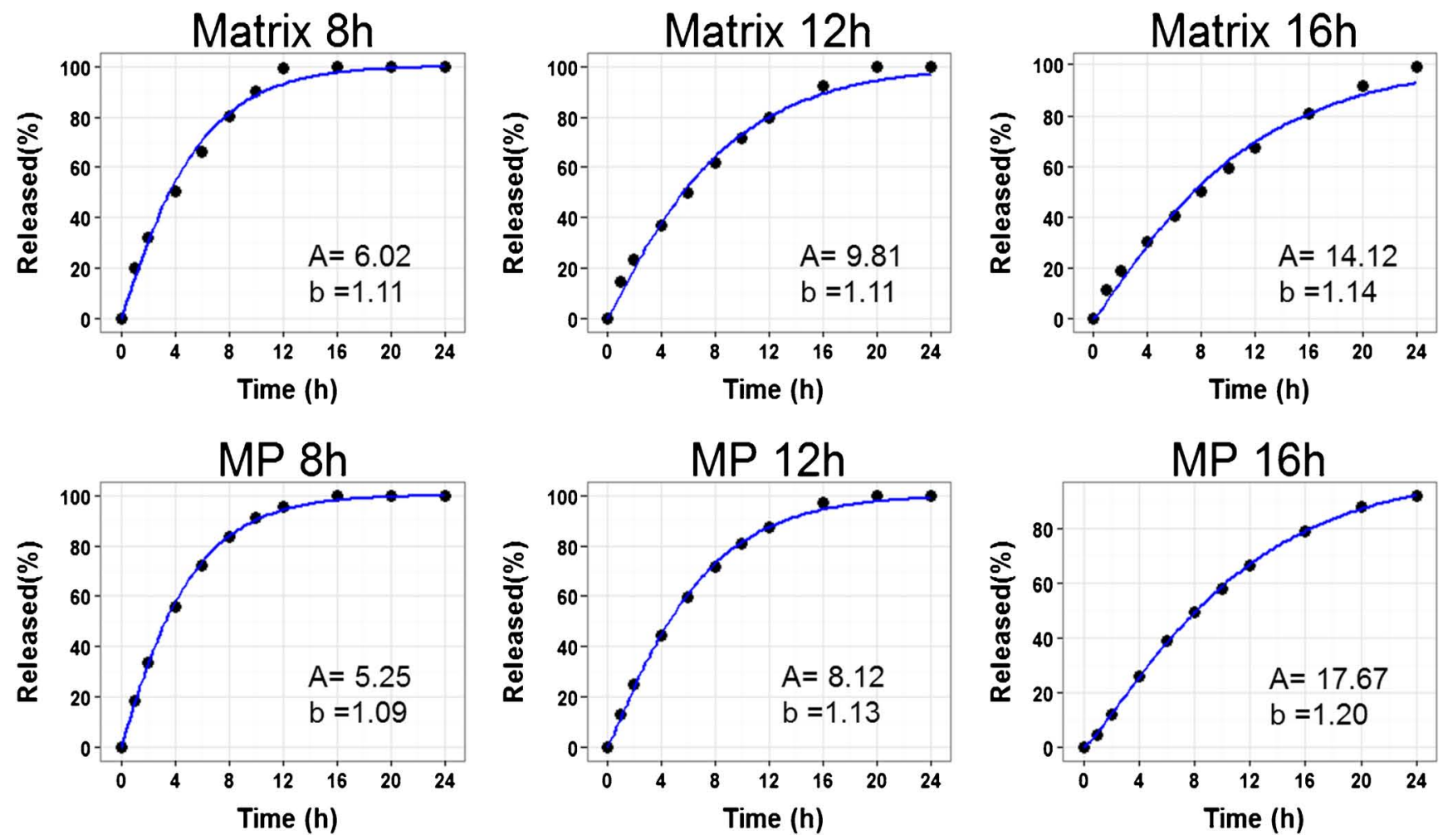

Fig. 5. Weibull fits for dissolution data used as input for the absorption PBPK IVIVC

traditional convolution-based IVIVC with PBPK-based IVIVC. To our knowledge, no publication is available that has attempted such evaluation based on a dedicated clinical study with two formulation technologies (matrix and multiparticulate MR systems).

The deconvolution-convolution-based approach is the most commonly used approach for development of IVIVCs for MR products. However, the deconvolution approach works better for compounds with fully linear pharmacokinetics. In cases where the total in vivo input appears to be dependent on the in vitro release rate (i.e., relative bioavailability of formulations differs), establishment of these linear models is not straightforward. Two publications have previously reported attempts to establish IVIVC for a compound with regiodependent absorption or differential bioavailability. Balan et al. (18) demonstrated that while a traditional convolution-based IVIVC was not successful for metformin, a compound known to exhibit regiodependent absorption, a successful IVIVC could be obtained by a modified approach that can be considered a convolution-based approach that allows for the model to take into account the differential bioavailability between formulations. More recently, Kakhi et al. (15) proposed a piecewise-linear variable absorption scale model, based on deconvolution-convolution approach, to obtain an IVIVC for a compound with differential bioavailability for one of the formulations. For MK-0941, we adopted a model similar to that reported by Kakhi et al. The model resulted in significant improvement in description of the in vivo absorption over time and a much improved correlation over a traditional time scale/shift model. The model generally adequately described the pharmacokinetic profile across the six formulations, although it should be acknowledged that depending on the model setup prediction errors did not fully meet the requirements for a regulatory submission (eg. while the multiparticulate only model passes all standard internal validation criteria, the matrix model fails the $C_{\max }$ criteria due to underprediction of the slow formulation). However at the early stages of formulation development that this study for MK-0941 was undertaken, the observed IVIVC would still be considered extremely useful in guiding the formulation development efforts.

Absorption/PBPK modeling is increasingly being used to guide formulation development. However there are relatively few reports specifically discussing the application of such models to MR products. Lukakova et al. (14) described the use of absorption models developed in GastroPlus to accurately predict the observed pharmacokinetics of MR formulations of metoprolol, a BCS I compound and of Adinazolam. In the latter case, the model was based on fit of the in vivo release rates to the pharmacokinetic data since in vitro release data were not available. For metoprolol, the authors were able to obtain a reasonable IVIVC by adjusting the colonic ASF values, similar to what was attempted in this manuscript. The authors concluded that even after this adjustment absorption of the slowest formulations was not fully predicted, however, overall prediction error was acceptable (14$20 \%$ ). Our simulations/IVIVC for MK-0941 indicates a very similar behavior; the slowest formulation of MK-0941 results in the highest prediction error $(\sim 20 \%$ prediction error similar to what was reported for metoprolol). In a more recent manuscript, Brown et al. (19) discussed the application of GastroPlus models to guide formulation development of a HIV-1 attachment inhibitor phosphate ester prodrug. The authors also employed a regiodependent adjustment on the ASF factors to describe the 

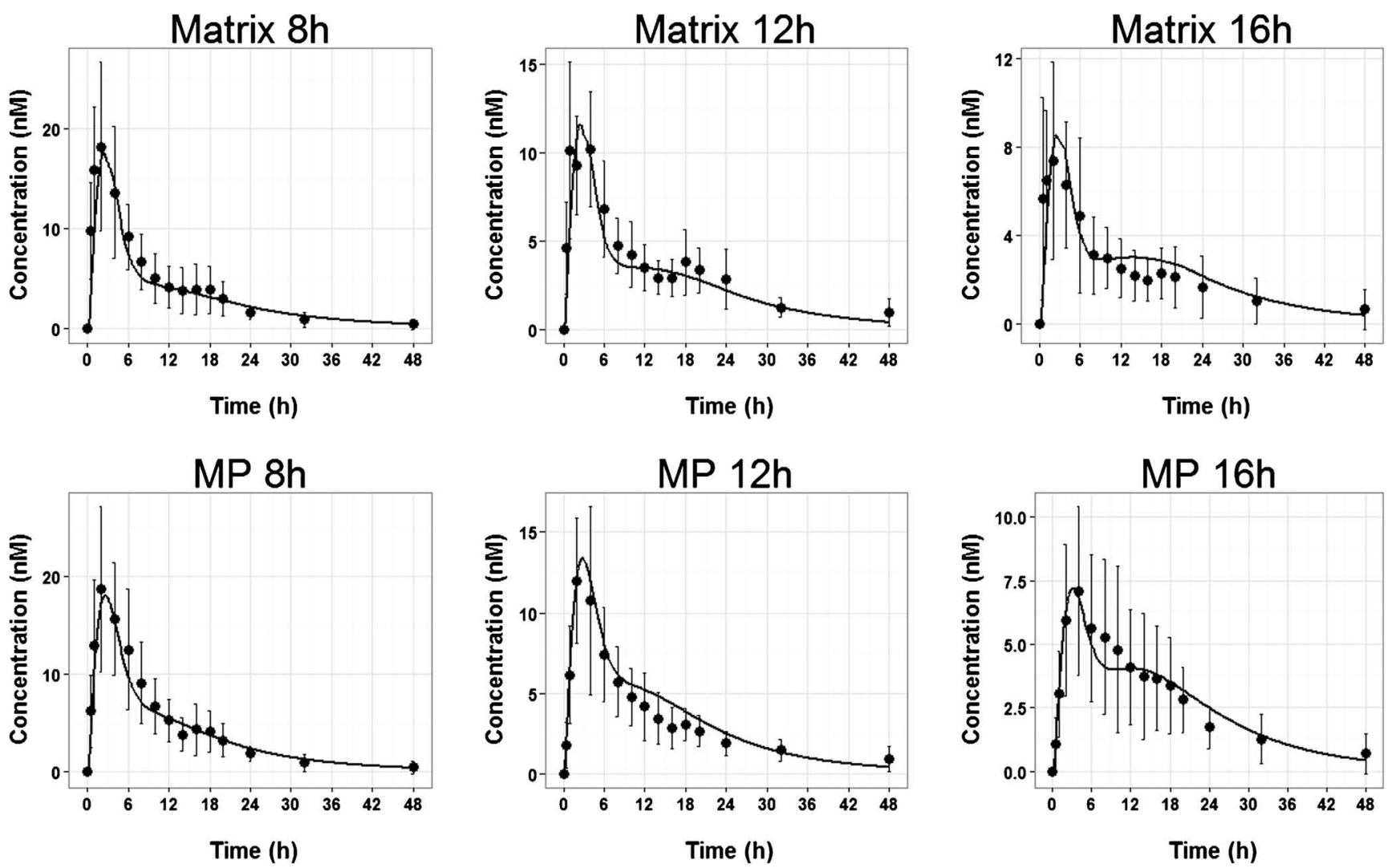

Fig. 6. Absorption PBPK IVIVC model predicted (lines) vs. observed (squares, mean \pm SD) plasma concentration $v s$. time profiles for MK-0941 ER formulation

absorption of the compound across the GI tract. While no IVIVC was attempted and assessment of prediction errors was not carried out, the simulation results from the authors provided same rank order with their projections.

In our study, we attempted to expand on these previous manuscripts by looking at the application of the ACAT model across six formulations developed by two different processes (three matrix and three multiparticulates). We further compared the predictions obtained from the absorption modeling efforts to the predictions that are obtained if a more traditional IVIVC is established. In general, we found that the two models resulted in comparable overall predictability and both models allowed for a good description of the overall formulation behavior. The traditional convolution-based IVIVC model required the implementation of the piecewise- linear variable absorption scale model to obtain best model description. The ACAT model required an adjustment of the ASF factors that could be seen as an analogous approach to allow for differential rate of absorption. The major difference between the models is that while for the traditional IVIVC model a correlation function between in vitro dissolution and in vivo absorption was implemented, the absorption model assumed that the in vitro dissolution is representative of in vivo release; the reasonable prediction of the pharmacokinetic data solely by adjustment of the ASF factor could be considered as an indication of a physiological reason for the differential behavior of the formulations rather than a disconnect between in vitro and in vivo release. We acknowledge that there are multiple other options to approach the absorption/PBPK IVIVC model. Ideally, a single set of ASF

Table V. Prediction Errors Using PBPK IVIVC Model

\begin{tabular}{|c|c|c|c|c|c|c|}
\hline \multirow[b]{2}{*}{ Formulations } & \multicolumn{3}{|l|}{$C_{\max }(\mathrm{nM})$} & \multicolumn{3}{|c|}{$\mathrm{AUC}_{0 \text {-last }}(\mathrm{nM} \times \mathrm{h})$} \\
\hline & Observed & Predicted & $\mathrm{PE}(\%)$ & Observed & Predicted & $\mathrm{PE}(\%)$ \\
\hline Matrix $8 \mathrm{~h}$ & 18.2 & 17.7 & -2.7 & 176 & 163 & -7.8 \\
\hline Matrix $12 \mathrm{~h}$ & 10.2 & 11.6 & 14.3 & 152 & 131 & -13.8 \\
\hline Matrix $16 \mathrm{~h}$ & 7.38 & 8.56 & 15.9 & 106 & 112 & 5.7 \\
\hline Matrix average & NA & NA & 9.14 & NA & NA & -5.28 \\
\hline Multiparticulate $8 \mathrm{~h}$ & 18.7 & 18.1 & -3.6 & 200 & 190 & -4.8 \\
\hline Multiparticulate $12 \mathrm{~h}$ & 12.0 & 13.4 & 11.4 & 152 & 168 & 10.4 \\
\hline Multiparticulate $16 \mathrm{~h}$ & 7.10 & 7.23 & 1.8 & 127 & 126 & -0.3 \\
\hline Multiparticulate average & NA & NA & 3.2 & NA & NA & 1.8 \\
\hline
\end{tabular}


values would be set across both matrix and multiparticulate formulations and a secondary correlation between in vitro and in vivo dissolution would be set separately for each one; these relationships may deviate from the 1:1 relationship used in the current model. In the absence of detailed studies mapping out the regional absorption of a compound (such data are not routinely generated during clinical development) to separately fit the ASF values, any model structure attempted on the MR formulations will result in interdependent estimates for ASF and in vitro-in vivo dissolution correlations that cannot be separately identified as unique values. Thus, we chose to present here the simplest approach where only the ASF values are fitted with the in vitro dissolution used as input. Despite not representing a unique solution, we believe our analysis indicates that absorption/ PBPK modeling IVIVC could be considered as an alternative to the traditional IVIVC approach to guide formulation development for MK-0941 at early stages

\section{CONCLUSIONS}

Establishment of an IVIVC is highly desirable during formulation development of MR products. We demonstrate that for a BCS III compound with regiodependent absorption, both a traditional convolution-based level A IVIVC and an absorption/PBPK-based IVIVC were able to adequately describe the compound pharmacokinetics to guide future formulation development. While there are limited reports on absorption/PBPK-based IVIVCs, this case study highlights the potential utility of this approach to complement more traditional IVIVC approaches for MR products.

\section{ACKNOWLEDGMENTS}

The authors would like to thank our colleagues from Merck Clinical Pharmacology, Pharmaceutical Sciences and Pharmacokinetics, Pharmacodynamics, and Drug Metabolism Departments who facilitated manufacturing of supplies, conduct of the clinical study and analysis of the plasma samples

\section{REFERENCES}

1. Guidance for industry: extended release oral dosage forms: development, evaluation, and application of in vitro/in vivo correlations, U.S. Department of Health and Human Services, Food and Drug Administration, Center for Drug Evaluation and Research (CDER), September 1997.

2. Guideline on the pharmacokinetic and clinical evaluation of modified release dosage forms (EMA/CPMP/EWP/280/96 Corr1) Draft XXIII, European Medicines Agency, 2013.
3. Draft guideline on quality of oral modified release products, European Medicines Agency, 2012.

4. Skelly J, Amidon G, Barr W, Benet L, Carter F, Robinson J, et al. Testing and correlation for oral controlled/modified-release dosage forms. Pharm Res. 1990;7(9):975-82.

5. Cardot JM, Beyssac E. In vitro/in vivo correlations: scientific implications and standardisation. Eur J Drug Metab Pharmacokinet. 1993;18(1):113-20.

6. Emami J. In vitro-in vivo correlation: from theory to applications. J Pharm Pharm Sci. 2006;9(2):169-89.

7. Dunne A, Gaynor C, Davis J. Deconvolution based approach for level A in vivo-in vitro correlation modeling: statistical considerations. Clin Res Regul Aff. 2005;22(1):1-14.

8. Gaynor C, Dunne A, Davis J. A comparison of the prediction accuracy of two IVIVC modelling techniques. J Pharm Sci. 2008;97(8):3422-32.

9. Kostewicz ES, Aarons L, Bergstrand M, Bolger MB, Galetin A, Hatley $\mathrm{O}$, et al. PBPK models for the prediction of in vivo performance of oral dosage forms. Eur J Pharm Sci. 2014;57:30021.

10. Agoram B, Woltosz WS, Bolger MB. Predicting the impact of physiological and biochemical processes on oral drug bioavailability. Adv Drug Deliv Rev. 2001;50 Suppl 1:S4167.

11. Jamei M, Turner D, Yang J, Neuhoff S, Polak S, RostamiHodjegan A, et al. Population-based mechanistic prediction of oral drug absorption. AAPS J. 2009;11(2):225-37.

12. Thelen K, Coboeken K, Willmann S, Dressman JB, Lippert J. Evolution of a detailed physiological model to simulate the gastrointestinal transit and absorption process in humans, part II: extension to describe performance of solid dosage forms. J Pharm Sci. 2012;101(3):1267-80.

13. Johnson KC. Dissolution and absorption modeling: model expansion to simulate the effects of precipitation, water absorption, longitudinally changing intestinal permeability, and controlled release on drug absorption. Drug Dev Ind Pharm. 2003;29(8):833-42.

14. Lukacova V, Woltosz WS, Bolger MB. Prediction of modified release pharmacokinetics and pharmacodynamics from in vitro, immediate release, and intravenous data. AAPS J. 2009;11(2):323-34.

15. Kakhi M, Marroum P, Chittenden J. Analysis of level A in vitro-in vivo correlations for an extended-release formulation with limited bioavailability. Biopharm Drug Dispos. 2013;34(5):262-77.

16. Kesisoglou F, Xie IH, Manser K, Wu Y, Hardy I, Fitzpatrick S. Suitability of a minipig model in assessing clinical bioperformance of matrix and multiparticulate extended-release formulations for a BCS class III Drug development candidate. J Pharm Sci. 2014;103(2):636-42.

17. Sutton SC, Evans LA, Fortner JH, McCarthy JM, Sweeney K. Dog colonoscopy model for predicting human colon absorption. Pharm Res. 2006;23(7):1554-63.

18. Balan G, Timmins P, Greene DS, Marathe PH. In vitro-in vivo correlation (IVIVC) models for metformin after administration of modified-release (MR) oral dosage forms to healthy human volunteers. J Pharm Sci. 2001;90(8):1176-85.

19. Brown J, Chien C, Timmins P, Dennis A, Doll W, Sandefer E, et al. Compartmental absorption modeling and site of absorption studies to determine feasibility of an extended-release formulation of an HIV-1 attachment inhibitor phosphate ester prodrug. J Pharm Sci. 2013;102(6):1742-51. 